\title{
STUDIES IN CONGESTIVE HEART FAILURE
}

\author{
XV. Reflex Versus Chemical Factors in \\ the Production of Rapid Breathing ${ }^{1}$ \\ By T. R. HARRISON, J. A. CALHOUN, G. E. CULLEN, \\ W. E. WILKINS AND C. PILCHER \\ (From the Departments of Medicine, Biochemistry, and Surgery, Vanderbilt \\ Medical School, Nashville)
}

(Received for publication August 7, 1931)

\section{INTRODUCTION}

Dyspnea in patients with cardiac disease may assume a number of different forms. The most important of these are:

$A$. Dyspnea upon exertion.

$B$. Dyspnea at rest:

1. Orthopnea,

2. Paroxysmal dyspnea (cardiac asthma),

3. Cheyne-Stokes respiration,

4. Continuous dyspnea.

The last four types of respiratory distress are likely to occur in various combinations with one another. Thus, a patient dying of congestive heart failure may feel severely short of breath at all times, even in the sitting position, but his dyspnea becomes worse when he attempts to assume the recumbent posture, and even when he sleeps sitting up, he wakens with violent paroxysmal exacerbations of the symptom. In such a patient, Cheyne-Stokes respiration is of ten present to further confuse an already extremely complex situation. Cheyne-Stokes respiration may occur without the simultaneous presence of the other forms of dyspnea. Orthopnea may also occur in "pure" form. Cardiac asthma is, however, invariably associated with orthopnea and of ten with Cheyne-Stokes respiration. Continuous resting dyspnea is almost always associated with one or more of the other forms of respiratory distress.

Do the various clinical forms of cardiac dyspnea have the same pathogenesis? It has been customary to say that they are all due to deficient cardiac output - a statement which is far from being proved by existing evidence. The fact that these several forms of dyspnea have distinctive clinical characteristics suggests that they are due to different physiological alterations, but this is also unproved.

${ }^{1}$ Aided by a grant from the National Research Council. 
It has seemed to us that any effort to reach an understanding of cardiac dyspnea should include

(a) a method of measuring respiratory distress,

(b) a study of each of the various types of dyspnea in "pure" form, and

(c) an investigation into the various combinations observed clinically.

A method of measuring respiratory distress has been developed and is reported in the tenth paper of this series (Harrison, Turley, Jones and Calhoun (1931)).

The dyspnea of mild exertion and that of orthopnea have been studied in regard to the relation of the dyspnea to the acid-base changes of the blood in paper thirteen (Cullen, Harrison, Calhoun, Wilkins and Timms (1931)) and paper fourteen (Calhoun, Cullen, Harrison, Wilkins and Tims (1931)). These studies showed that neither of these types of dyspnea is due to chemical changes in the arterial or venous blood, or to diminished cerebral blood flow.

The respiratory center may be influenced in at least three general ways: (a) By chemical changes in the blood, (b) by impulses from the cerebral cortex, $(c)$ by reflexes. Having demonstrated that alterations. of the first of these influences cannot explain either orthopnea or dyspnea on exertion, and considering it extremely unlikely that any type of dyspnea except that observed in hysteria and allied states can be of psychic origin, we have been led to the conclusion, by exclusion, that the explanation for the types of respiratory distress under discussion was to be sought in reflex disturbances. Because of the fact that functional alterations, i.e., diminished vital capacity, etc., in the lungs are invariably present in patients with cardiac failure, and since it is well known that respiratory reflexes do arise in the lungs, it was felt that a study of the effects of experimental diminution in vital capacity on breathing was indicated.

No attempt is made here to review all of the voluminous literature concerning the reflex regulation of breathing; only a few of the investigations which are of particular importance for the present work will be cited. More than a century ago, Legallois (1812) observed that cutting the vagus nerves caused marked decrease in rate and increase in depth of breathing. Herring and Brauer (1868) showed that distention of the lungs with air caused an expiratory movement. These effects, which were absent after cutting the vagus nerves, were interpreted as indicating that the rate of breathing was controlled by alternating excitant and inhibitory impulses from the lungs to the respiratory center by the vagus nerves. Heymans and Heymans (1927) demonstrated that, in addition to these "mechanical" reflexes from the lungs, there existed also, through the vagi, afferent impulses to the respiratory center from the heart and aorta, and that so long as the vagus nerves were intact respiration could be affected by alterations of the pressure and composition of the blood in the heart and aorta. Hertzman and Gesell (1927) also found evidences for reflexes from the heart and aorta, but felt that these were less important and less constant than the mechanical reflexes from the lungs. 
Moore (1927) showed that vagal afferent respiratory reflexes were not solely dependent on lung motion. He separated the breathing of the two lungs by cannulating the two primary bronchi. If, when a lung contained oxygen, its bronchus were blocked, gradual acceleration of breathing developed, this being sometimes temporary but usually sustained. Cutting the vagus nerve on the corresponding side led to a prompt slowing of the breathing. These researches were extended by Moore and Harrison in work which has not been published. These authors concluded that atelectasis was an important cause of tachypnea, this opinion being based upon the following evidence.

1. If a lung with intact circulation be filled with oxygen and its bronchus blocked, atelectasis develops and a gradual increase in respiratory rate occurs.

2 . If the experiment be repeated with the corresponding pulmonary artery ligated, neither atelectasis nor tachypnea develops.

3. If the lung contains nitrogen and has an intact circulation, blocking its bronchus does not cause atelectasis or tachypnea.

4. Cutting either vagus nerve slowed the respiratory rate, when tachypnea had been produced.

Moore and Harrison (unpublished data) interpret these observations as meaning that the atelectatic lung was responsible for sending more or less continuous inspiratory impulses to the respiratory center, whereas the other lung, being the sole functioning lung, underwent inflation more rapidly than normal, and soon reached a sufficient degree of distention to release inhibitory (expiratory) impulses to the center. The result was rapid and shallow breathing, which was to be regarded as essentially due to incoordination of the vagal reflexes.

Harrison and Moore (1928), in another study, found that the vagal influences on depth and on rate of breathing were of ten dissociated. If one bronchus were compressed so that the corresponding lung had a considerably smaller respiratory excursion than its fellow, cutting the vagus on the corresponding side had little or no effect on respiratory rate but was always associated with a pronounced increase in respiratory depth. As a result of these and similar observations Harrison and Moore (1928) suggested that the movement of the lungs was the predominant factor in the reflex control of respiratory rate, whereas some other factor, possibly reflexes from the heart and aorta, was especially important in the reflex control of respiratory depth.

It is to be noted that previous students of the subject have not usually made complete studies of the blood gases and hydrogen ion concentration in their observations on reflex regulation of breathing. Consequently there remains the remote possibility that such alterations as were found might have been due to chemical changes in the blood. Gesell (1927) has emphasized the importance of the blood flow through the respiratory center in the control of respiration and has pointed out that in order to draw conclusions concerning the influence of the blood on the center it is necessary to know the composition of the blood coming from as well as that going to the brain. In the present study we have analyzed both arterial and cerebral venous blood.

\section{METHODS}

Dogs were used. Sodium barbital in doses of approximately $0.3 \mathrm{~cm}$. per kilo of body weight was given two to three hours before the experiment. Tracheotomy was done, and one femoral artery was exposed. The large occipital venous sinus, which has been described by Pilcher (1930) and which drains most of the blood from the posterior portions of 
the brain stem, was exposed. Throughout the course of the experiment the animal breathed into a Benedict spirometer, which was filled with oxygen, the carbon dioxide being absorbed in the usual way. Respiratory rates were counted from the tracing except when the breathing was very rapid, in which instance the respirations were counted minute by minute in the course of the observations.

Control observations were made of the respiratory rate and samples of arterial and of occipital venous blood were taken. The vital capacity was then decreased either by (1) introducing air into the thoracic cavity; (2) running Ringer's solution into the lungs through the tracheal cannula, or (3) distending the capillaries of one lung with blood from another dog. The latter procedure was performed as follows.

Before starting the experiment the left fourth or fifth rib was resected, the pleura opened, and artificial respiration instituted. A small glass cannula connected to a small bore rubber tube was then tied into the left pulmonary artery, the open end of the cannula pointing toward the lung. The left pulmonary artery proximal to the cannula was ligated. The left pulmonary veins were then tied. The chest was closed around the rubber tube, care being taken to expel all possible air from the pleural cavity and to distend the lungs with the air pump in order to reinflate any portions which had become atelectatic as a result of the operation. Artificial respiration was then discontinued. At least one half hour was allowed for the breathing to become stabilized before the experimental procedure was begun. In a few instances the animals breathed very rapidly after operation and morphine was given to slow the breathing before the control studies were made. By this technique it was possible to produce any desired degree of congestion of one lung at will, as blood could be introduced into the lung and could not escape from it.

It is obvious that although all of these three procedures reduce the vital capacity, it is certain that introduction of fluid either into the alveoli or vascular bed of the lung has additional reflex effects which are not produced by pneumothorax. In this study, however, it is evident that the reduction of the vital capacity is the important common factor and discussion of the other effects will be omitted during the remainder of this paper for the sake of simplicity.

After the vital capacity had been reduced by one of the methods described the breathing became rapid and a second pair of blood samples (arterial and occipital venous) were taken. The vagus nerves were then cut and the entire experimental procedure repeated. The experiments varied somewhat in the exact details, which are shown in the tables.

The blood samples were taken under oil with Luer syringes. Contamination with air did not occur. Approximately six cubic centimeters of blood were expelled under oil into a bottle containing oxalate. This was used for oxygen determination, which was performed on the Van Slyke- 
Neill (1924) apparatus. Ten to fifteen cubic centimeters of blood were placed under oil in a Pyrex centrifuge tube, the oil was replaced with melted paraffin, and immediately centrifuged. The serum was used for determinations of $\mathrm{pH}$ by Cullen's method (1922), and of carbon dioxide content as described by Van Slyke and Sendroy (1927). Carbon dioxide tensions were calculated by the Henderson-Hasselbalch formula, a value of 6.1 being assumed for $\mathrm{pK}^{\prime}$. No claim is made for absolute accuracy of the $\mathrm{pH}$ or $p \mathrm{CO}_{2}$ values for the reason that the correction factor $(\mathrm{C})$ of dog's blood for changing colorimetric to electrometric values varies with individual dogs. In our calculations no correction was used since we were interested in changes in $\mathrm{pH}$. Likewise the values for $p \mathrm{CO}_{2}$ are comparative. Values for carbon dioxide content are accurate to within 0.2 volume per cent and values for oxygen to within 0.3 volume per cent.

\section{RESULTS}

\section{A. Changes in the rate and depth of breathing}

The findings are shown in Table 1 and in Figures 1,2 and 3. The control respiratory rates varied between 3 and 48 . In those experiments in which the thoracic cavity had been opened the rate of breathing was usually more rapid than when this had not been done.

When the vagus nerves were intact diminution in vital capacity, by any of the three methods used, always resulted in increased respiratory rate, although the degree of increase varied in different animals. The depth of respiration was decreased. The effect of decreasing the vital capacity on the minute ventilation was variable. In the majority of the experiments, in which the vagus nerves were intact, the minute ventilation was increased, although in three instances no significant change was noted and in one experiment a marked diminution occurred. In several instances pneumothorax was followed by an initial increase in ventilation and later, when still more air had been injected into the pleural cavity, the ventilation diminished. In general it may be said that, when the vagus nerves are intact, slight decrease in vital capacity results in moderate increase in the rate and slight decrease in the depth of breathing with some increase in the ventilation per minute, whereas marked decrease in vital capacity results in marked increase in respiratory rate and marked decrease in respiratory depth so that the minute ventilation may be either greater or less than the normal for the animal.

Cutting the vagus nerves was followed by a marked decrease in the rate of breathing in all experiments save one. In this instance the respiratory rate was already very slow and became slightly faster after vagotomy. In every experiment, including the one just mentioned, bilateral vagotomy resulted in marked increase in depth of breathing. As has been pointed out, Harrison and Moore (1928) have already shown that the vagal influ- 

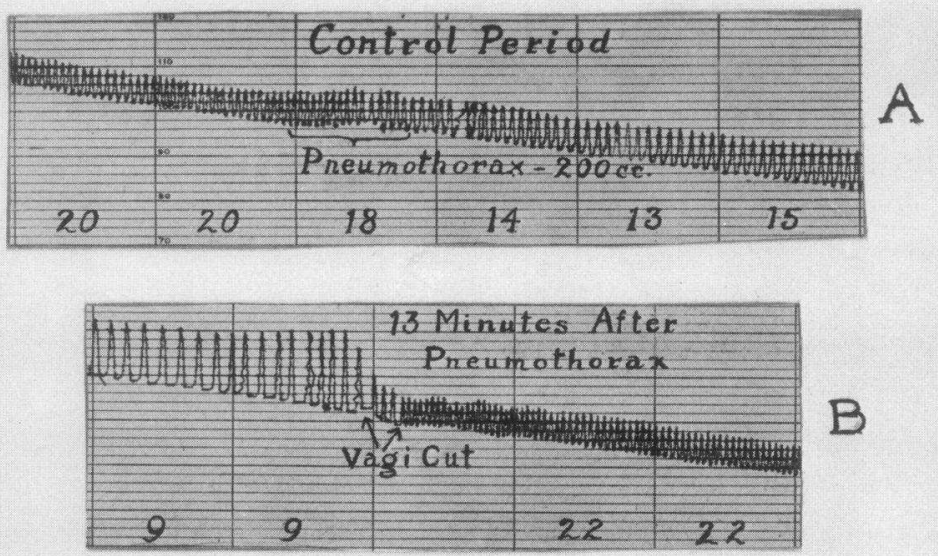

B
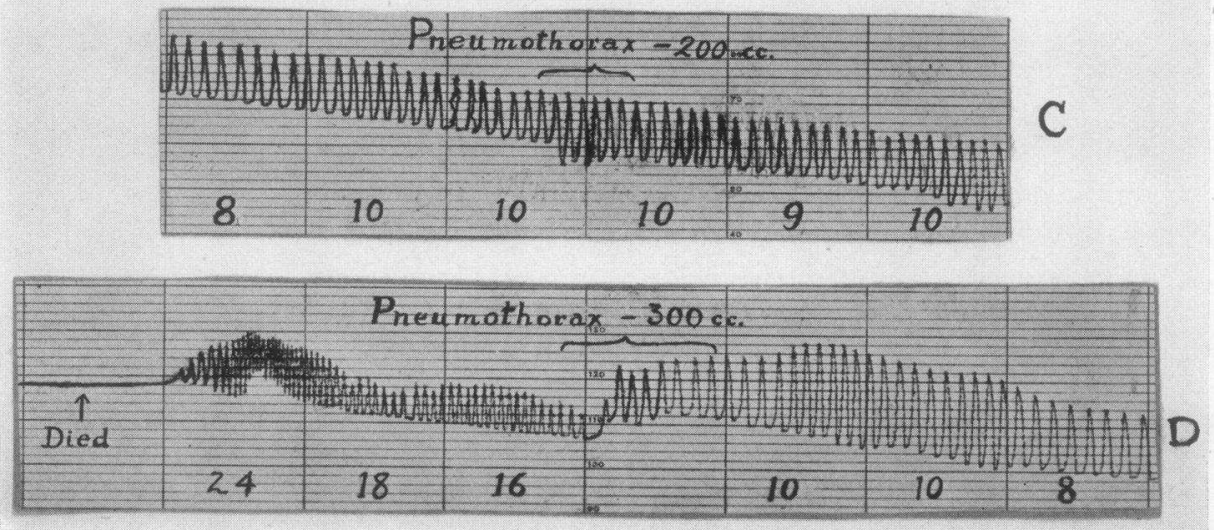

Fig. 1. The Curves Run from Right to Left

The numbers refer to respiratory rate per minute. Production of pneumothorax when the vagus nerves were intact $(A)$ was followed by a moderate increase in respiratory rate. This persisted until the vagus nerves were cut $(B)$. After vagotomy, pneumothorax did not increase the rate of breathing $(C)$ until sufficient air was introduced to seriously decrease the depth of breathing. Then there was marked tachypnea followed by apnea and death $(D)$. Significant chemical changes in the blood did not occur until the final introduction of air in to the chest. 


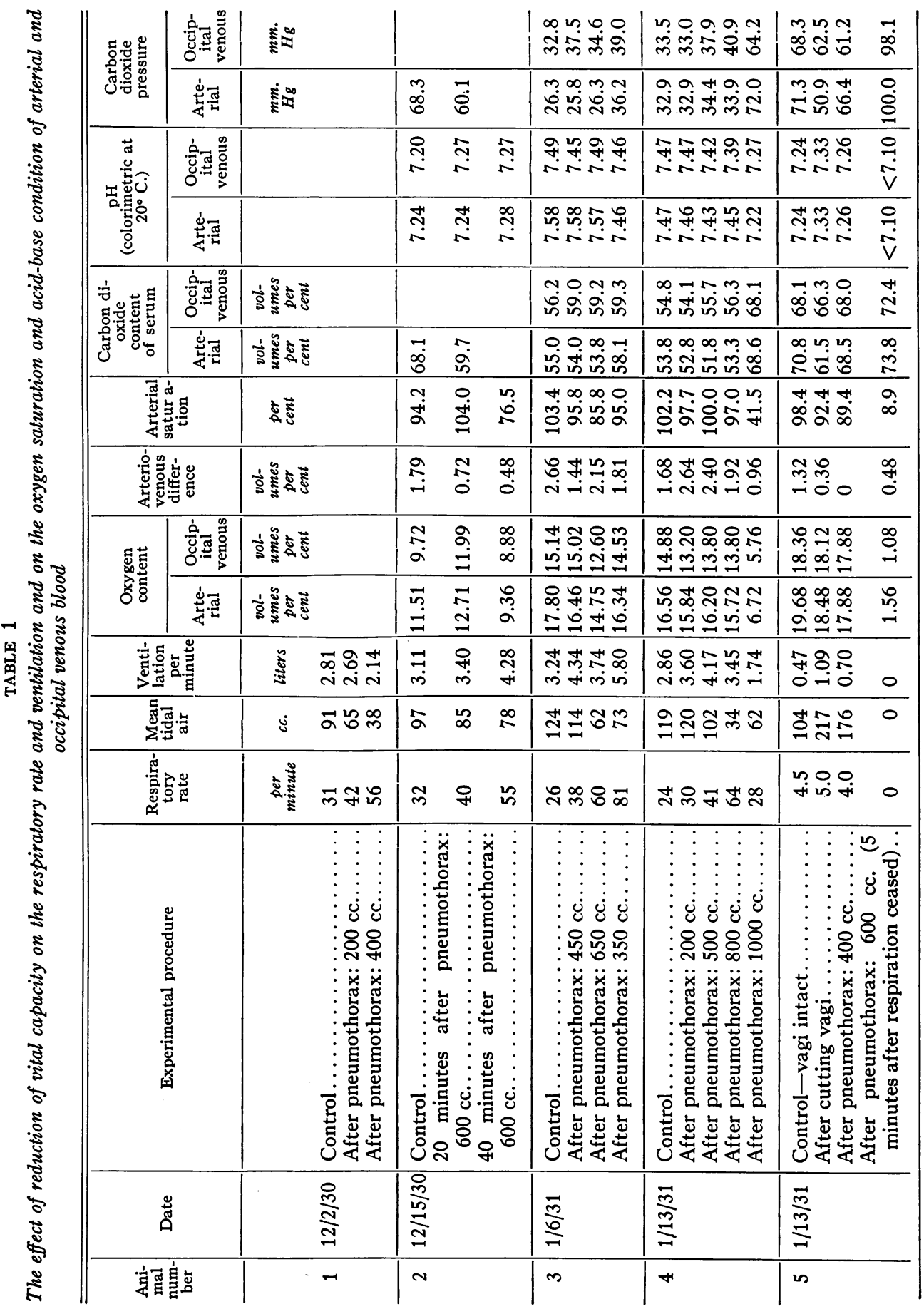




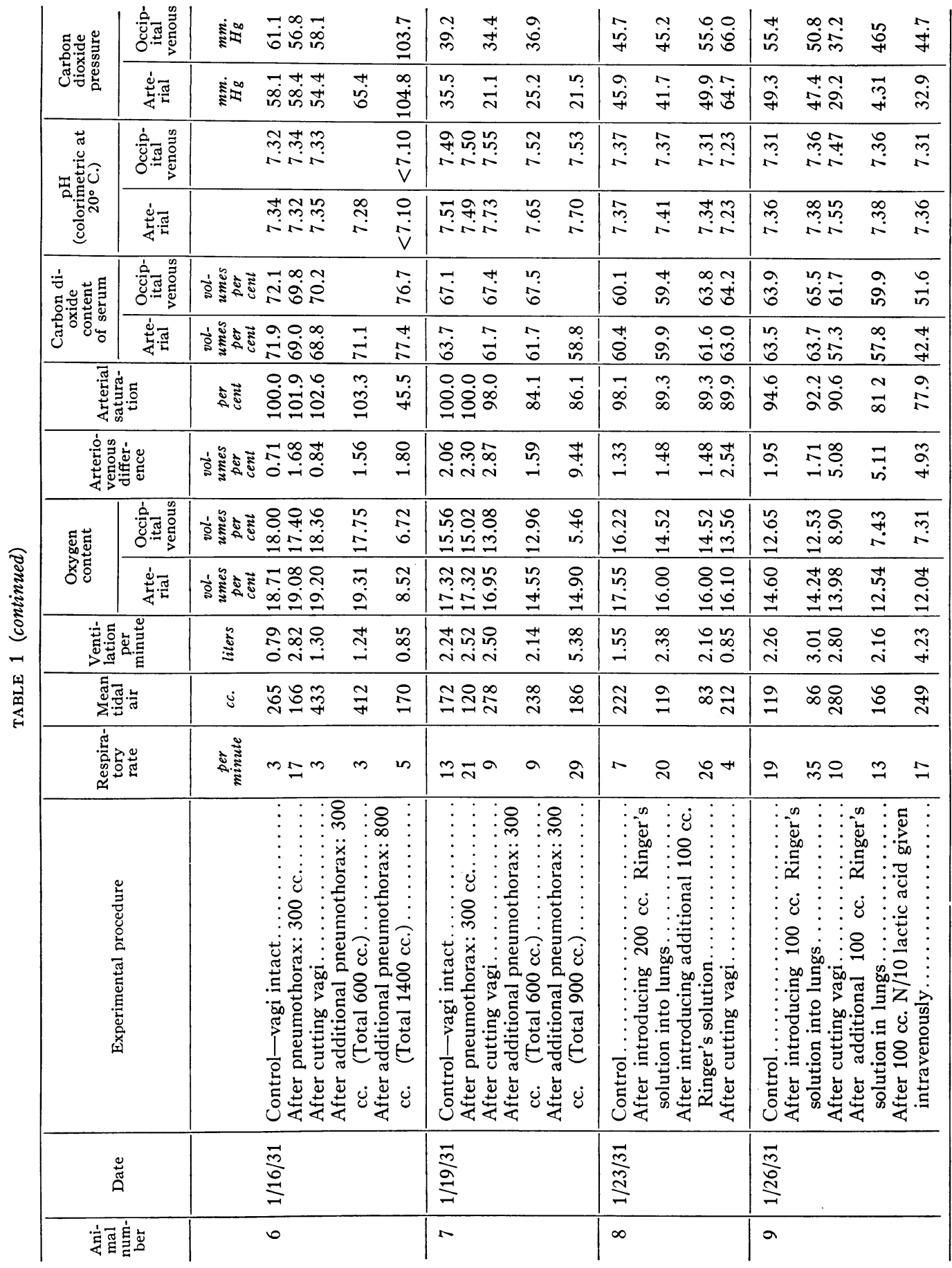




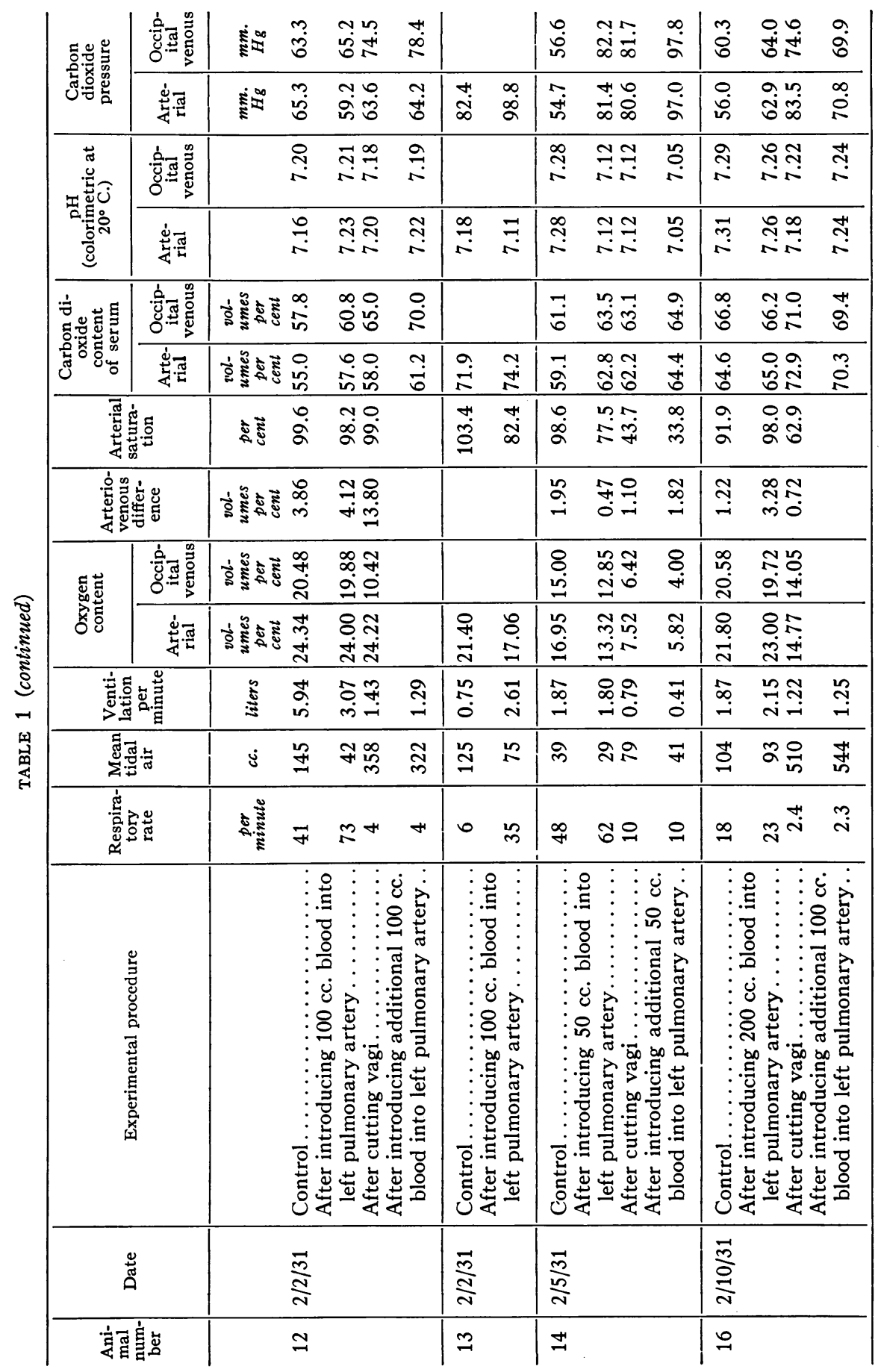


ence on respiratory depth may be present when there is no influence on respiratory rate.

Vagotomy usually caused decrease in minute ventilation, although several experiments were exceptional in this regard. After the vagi had been cut, decrease in vital capacity caused increase in the respiratory rate in only two of seven experiments and in one of these the degree of increase

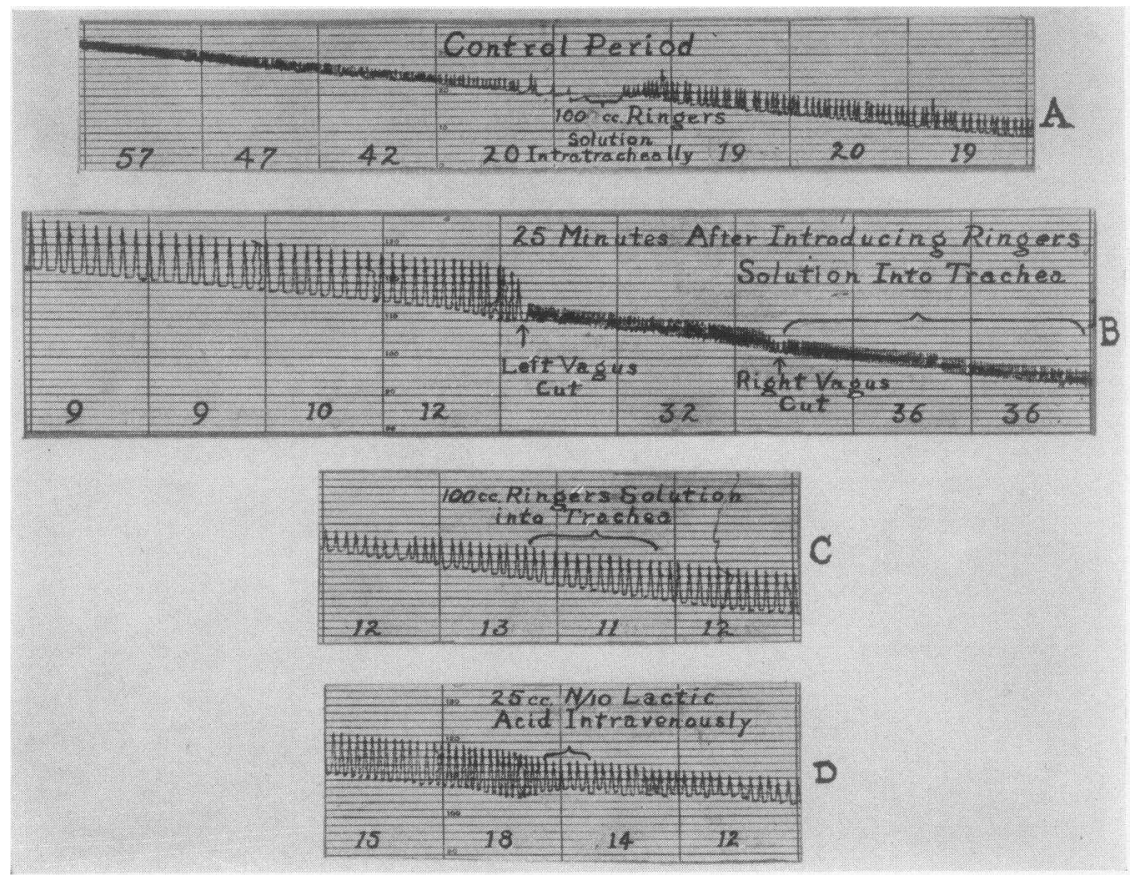

Fig. 2. The Curves Run from Right to Left

The introduction of Ringer's solution into the lungs caused, after a short period of apnea, marked acceleration of breathing when the vagus nerves were intact $(A)$. Cutting the vagi $(B)$ slowed the breathing. After vagotomy, Ringer's solution was again introduced into the lungs but did not cause rapid breathing $(C)$. However, the animal was able to respond to chemical stimuli as was shown by injecting lactic acid $(D)$.

was small. In the remaining five observations the same procedures which invariably caused rapid breathing in animals with intact vagus nerves had no effect on the respiratory rate. (The reasons why two experiments were exceptions to the general rule are given below.) In vagotomized animals, reduction in vital capacity by any of the three methods used was usually followed by diminution in the depth of breathing and in the minute ventilation. 


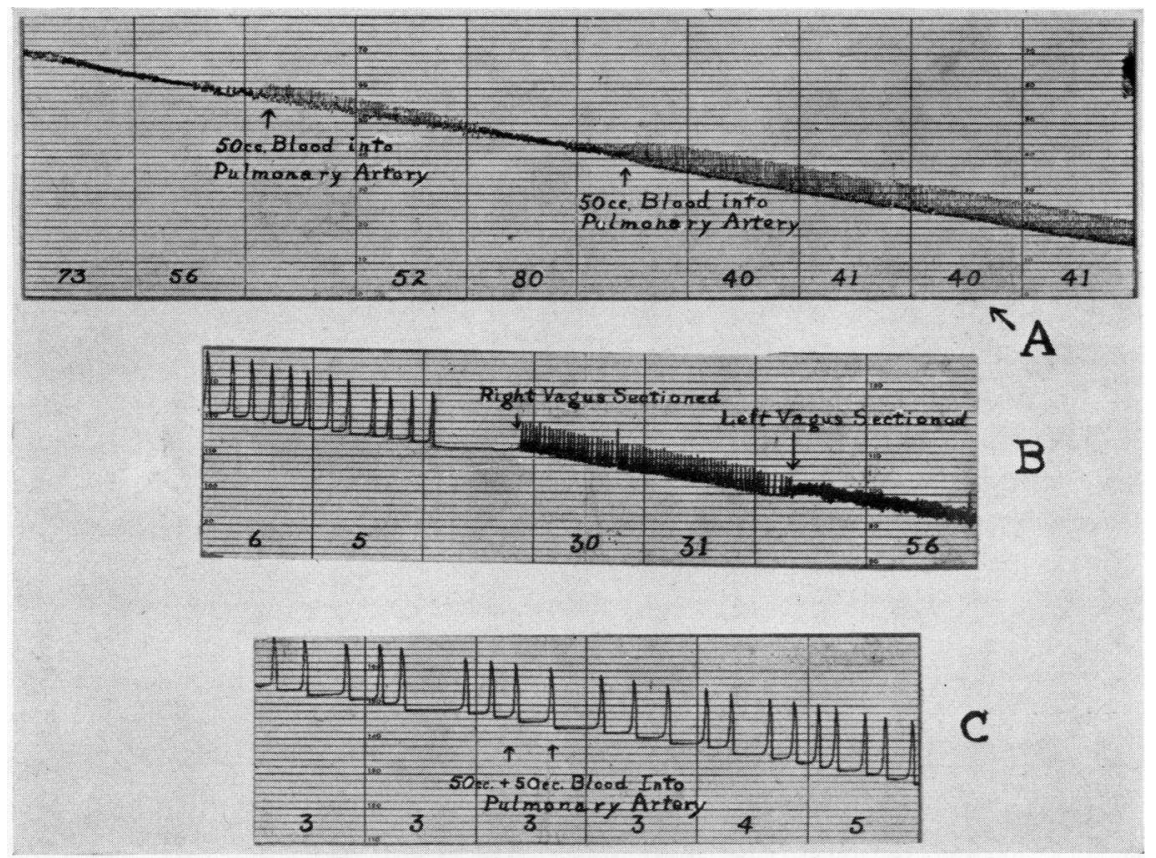

Fig. 3. The Curves Run from Right to Left

When the vagus nerves were intact distention of the pulmonary capillaries with blood caused tachypnea $(A)$. Cutting the vagi caused slowing of the breathing $(B)$. After vagotomy, introducing additional blood into the lung did not increase the rate of breathing $(C)$.

\section{B. Changes in the oxygen saturation and acid-base condition of the blood}

The initial arterial saturation with oxygen was usually high, as the animals were breathing oxygen. In only two of twelve observations was there significant decrease in the arterial saturation at the time when the respirations first increased in rate. In animals with the vagi intact, progressive diminution in vital capacity caused tachypnea before significant oxygen unsaturation developed. With marked decrease in vital capacity there is a decreased oxygen saturation of arterial blood. The arterial unsaturation therefore could not have been the cause of the tachypnea. Likewise, significant alterations in occipital venous oxygen content did not occur from slight reduction in vital capacity. The arteriovenous difference did not undergo any constant changes and hence the rapid breathing cannot be ascribed to diminished cerebral blood flow.

The onset of tachypnea was usually associated with slight diminution of the carbon dioxide content of the blood, both arterial and occipital venous, although in several experiments slight rise in carbon dioxide content was observed. The degree of change was usually not striking. Consistent alterations in $\mathrm{pH}$ were not found. In some experiments no change was 
noted, in others a decrease, and in still others an increase occurred at the time when rapid breathing began. Likewise, consistent alterations in $p \mathrm{CO}_{2}$ did not occur. In the majority of instances decrease in vital capacity caused an initial reduction in carbon dioxide pressure but at times the reverse was found.

Cutting the vagus nerves was usually followed by no marked change in the arterial oxygen saturation. However, in two experiments the marked slowing of respiration was associated with a sharp decrease in arterial saturation. Vagotomy usually altered the occipital venous saturation. The arteriovenous oxygen difference between the blood entering and that leaving the brain was decreased (indicating greater blood flow) three times and increased (indicating lowered blood flow) five times. The differences observed after vagotomy were usually well outside the errors of the method. It appears probable, therefore, that cutting the vagus nerves may be followed either by increase or decrease in cerebral blood flow.

The carbon dioxide content and the $\mathrm{pH}$ of the blood were not altered in any constant direction by vagotomy. When the respiratory rate before vagotomy was extremely slow, cutting the vagus nerves resulted in greater ventilation and was likely to be followed by diminution in the carbon dioxide tension and content of the blood and by increase in $\mathrm{pH}$ (Experiment 5). In other instances, when vagotomy produced extreme slowing of the respiration, the reverse change occurred (Experiment 16). Often, cutting the vagi produced no significant change in the carbon dioxide content or hydrogen ion concentration, despite marked changes in the rate and character of the breathing.

In the majority of instances the same procedures which produced no consistent changes in dogs with intact vagi were followed by changes in the direction of carbon dioxide acidosis in vagotomized dogs. In the latter animals diminution of vital capacity was as.a rule followed by increase in carbon dioxide content, decrease in $\mathrm{pH}$ and marked increase in carbon dioxide tension of the serum.

Taken as a whole, such chemical changes as were found in the blood were clearly the results and not the causes of the changes observed in ventilation. This conclusion is the same as that arrived at in our studies on ventilation and blood composition in patients with cardiac disease.

\section{The effect of changes of oxygen saturation and acid-base condition of the blood on breathing}

In a number of the experiments which have been mentioned changes in the composition of the blood were found. These were usually slight and it was not believed that changes of such small magnitude could account for the alterations observed in breathing. However, in order to be more certain of this point, experiments were undertaken to determine 
the sensitivity of dogs anesthetized in the same manner to changes in the gases and $\mathrm{pH}$ of the blood. The data are shown in Table 2 and Figures 4 and 5 .

Anoxemia was produced by allowing the animals to rebreathe air from a Benedict spirometer, the carbon dioxide being absorbed. In each instance increase in ventilation resulted, this being due either to increase

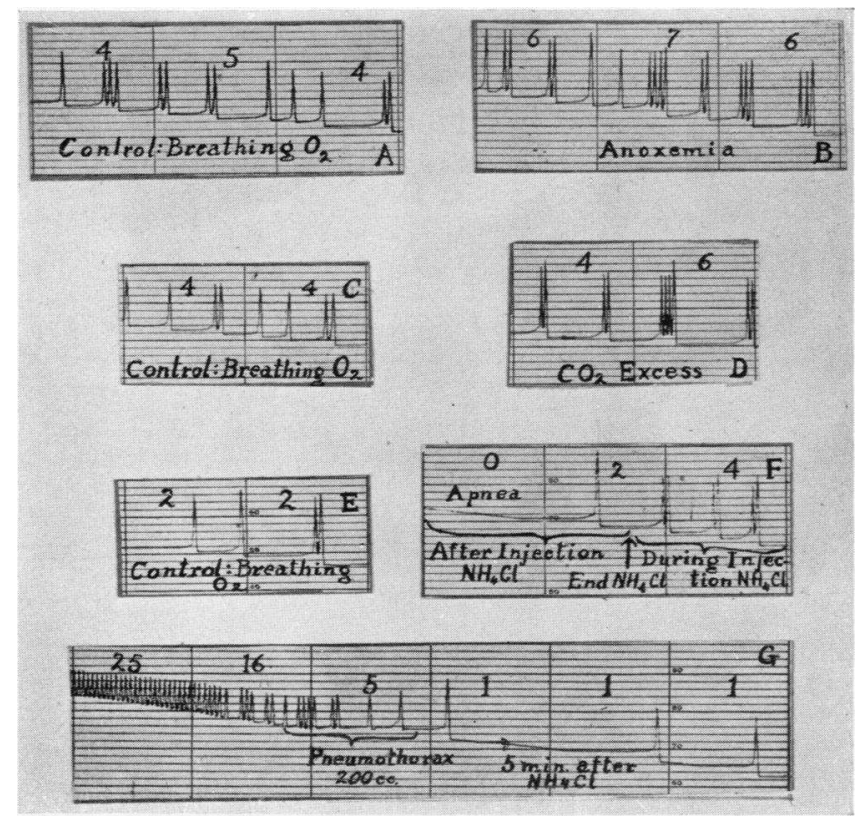

Fig. 4. The Curves Run from Right to Left

The vagus nerves were intact throughout the experiment. Anoxemia $(B)$ caused increase in rate and depth as compared to the control period $(A)$. Carbon dioxide excess $(D)$ caused increased depth and slight increase in rate of breathing as compared to the control period $(C)$. Intravenous injection of ammonium chloride $(F)$ caused increase in respiratory rate and depth, as compared to the breathing before the injection $(E)$. Following the injection of ammonium chloride apnea developed $(F)$. This was followed by extremely slow breathing $(G)$. Pneumothorax caused prompt and marked increase in the respiratory rate with diminution in depth $(G)$. The experiment illustrates the greater sensitivity of the respiratory rate to changes in the vital capacity (reflex stimuli) than to change in the composition of the blood (chemical stimuli).

in rate, increase in depth, or both. In no instance did anoxemia result in extreme tachypnea. It is therefore clear that the changes in respiratory rate which occurred in the previous experiments when the vital capacity was reduced could not have been due to the slight degrees of anoxemia which occasionally developed.

It appears from Table 2 that, in order to double the minute ventilation the arterial saturation must be reduced to sixty per cent or less. Oxygen 
Vagi Intact

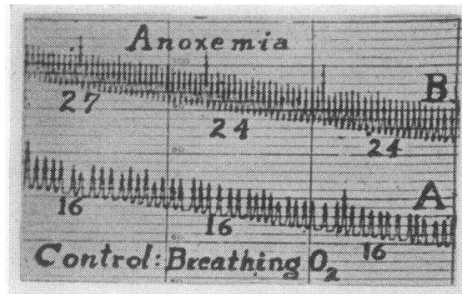

Vagi Cut
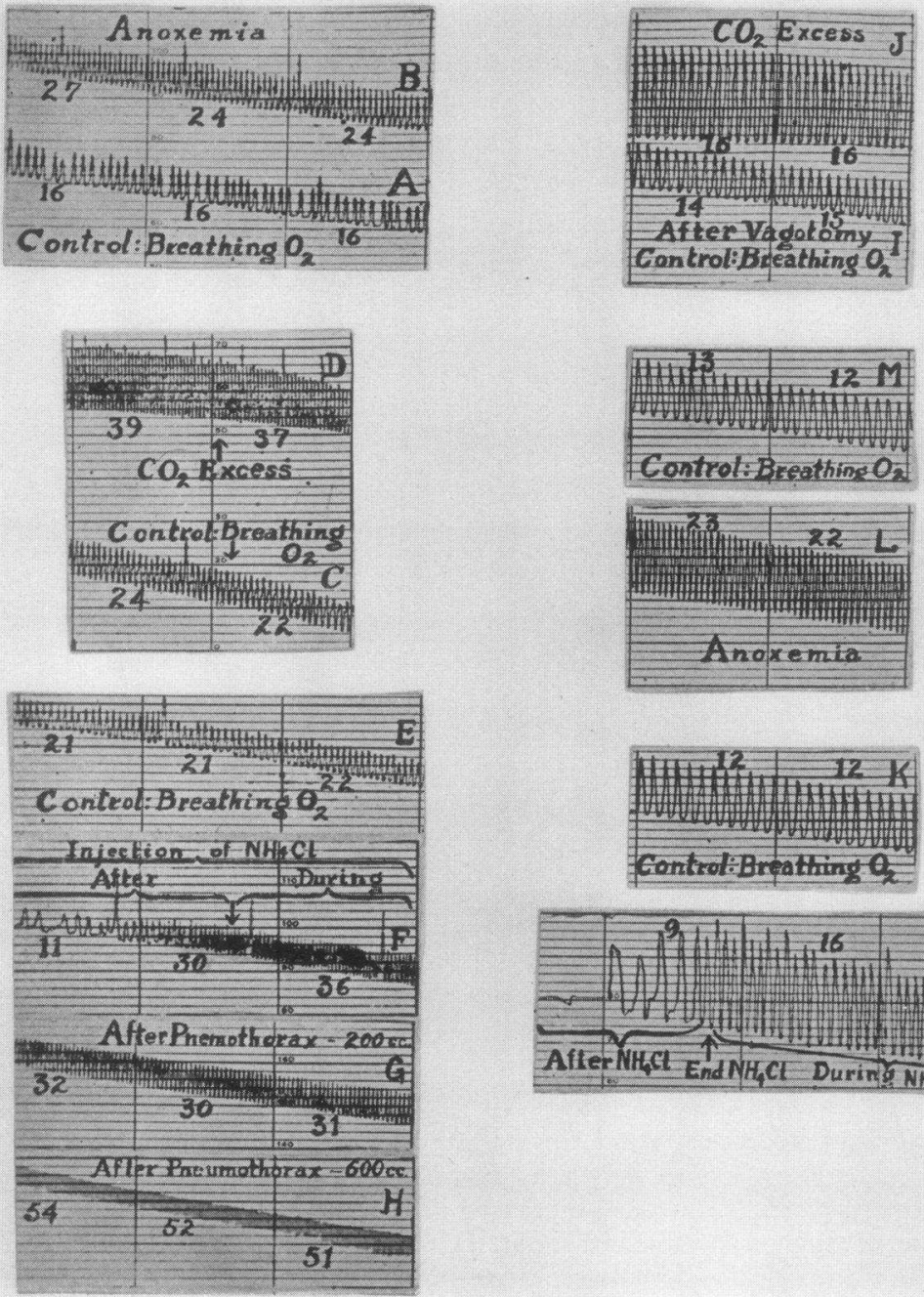

Fig. 5. The Curves Run from Right to Left

Oxygen lack $(B)$ caused moderate increase in respiratory rate and slight increase in respiratory depth. Carbon dioxide excess $(D)$ caused moderate increase in rate and marked increase in depth of breathing. Intravenous administration of ammonium chloride $(F)$ caused increase in respiratory rate with no distinct change in depth. Following the injection the rate of breathing diminished markedly $(F)$. A small amount of air in the pleural cavity caused moderate increase in respiratory depth $(G)$ and further pneumothorax caused extreme tachypnea $(H)$. Cutting the vagus nerves diminished the rate and increased the depth of breathing $(I)$. After vagotomy carbon dioxide excess $(J)$ produced marked increase in depth and slight increase in rate, oxygen lack caused increase in both rate and depth $(L)$, whereas ammonium chloride caused hyperpnea followed by apnea $(N)$. The response to chemical changes is similar whether the vagus nerves are cut or intact, whereas the response to diminished vital capacity is dependent on the vagus nerves. 
TABLE 2

The effect on respiration of changes in oxygenation and acid-base condition of the arterial blood of dogs in relation to vagotomy

\begin{tabular}{|c|c|c|c|c|c|c|c|c|c|c|}
\hline $\begin{array}{c}\text { Ani- } \\
\text { mal } \\
\text { num- } \\
\text { ber }\end{array}$ & Experimental procedure & $\begin{array}{c}\mathrm{O}_{2} \\
\text { con- } \\
\text { tent }\end{array}$ & $\begin{array}{c}\mathrm{O}_{2} \\
\text { satu- } \\
\text { ration }\end{array}$ & $\begin{array}{l}\mathrm{CO}_{2} \\
\text { con- } \\
\text { tent of } \\
\text { serum }\end{array}$ & $\begin{array}{c}\mathrm{pH} \\
\text { of } \\
\text { serum }\end{array}$ & $\begin{array}{c}\mathrm{CO}_{2} \\
\text { ten- } \\
\text { sion of } \\
\text { serum }\end{array}$ & $\begin{array}{l}\text { Respir- } \\
\text { atory } \\
\text { rate }\end{array}$ & $\begin{array}{c}\text { Mean } \\
\text { tidal } \\
\text { air }\end{array}$ & $\begin{array}{l}\text { Venti- } \\
\text { lation } \\
\text { per } \\
\text { minute }\end{array}$ & Remarks \\
\hline 17 & 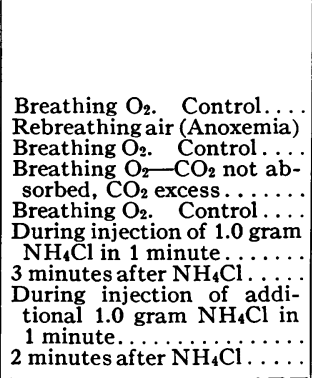 & $\begin{array}{c}\text { vol- } \\
\text { umes } \\
\text { per } \\
\text { cent } \\
19.32 \\
15.60 \\
19.20 \\
19.91 \\
19.32\end{array}$ & $\begin{array}{r}\text { per } \\
\text { cent } \\
\\
100.6 \\
81.3 \\
100.0 \\
\\
103.7 \\
100.6\end{array}$ & $\begin{array}{l}\text { vol- } \\
\text { umes } \\
\text { per } \\
\text { cent } \\
48.2 \\
41.4 \\
45.5 \\
\\
52.3 \\
47.6\end{array}$ & $\begin{array}{l}7.55 \\
7.75 \\
7.60 \\
7.48 \\
7.52\end{array}$ & $\begin{array}{l}\mathrm{mm} . \\
\mathrm{Hg} \\
\\
24.6 \\
13.5 \\
20.8 \\
31.2 \\
25.9\end{array}$ & \begin{tabular}{|c|} 
per \\
minute \\
\\
33 \\
38 \\
18 \\
25 \\
21 \\
43 \\
1
\end{tabular} & $\begin{array}{r}c c . \\
\\
87 \\
120 \\
114 \\
166 \\
124 \\
120 \\
210\end{array}$ & $\begin{array}{l}\text { liters } \\
2.87 \\
4.57 \\
2.05 \\
4.15 \\
2.61 \\
5.16 \\
5.16 \\
\\
6.96 \\
0.18\end{array}$ & $\begin{array}{l}\text { Vagi } \\
\text { intact } \\
\text { through- } \\
\text { out } \\
\text { experi- } \\
\text { ment }\end{array}$ \\
\hline 18 & $\begin{array}{l}\text { Breathing } \mathrm{O}_{2} . \text { Control .... } \\
\text { Rebreathing air (Anoxemia) } \\
\text { Breathing } \mathrm{O}_{2} . \text { Control } \ldots . . \\
\text { Breathing } \mathrm{O}_{2}-\mathrm{CO}_{2} \text { not } \mathrm{ab}- \\
\text { sorbed, } \mathrm{CO}_{2} \text { excess } \ldots \ldots \ldots \\
\text { Breathing } \mathrm{O}_{2} \text {. Control } \ldots \ldots \\
\text { During injection of } 1.0 \text { gram } \\
\mathrm{NH}_{4} \mathrm{Cl} \text { in } 4 \text { minutes } \ldots \ldots \\
\text { After injection of } \mathrm{NH}_{4} \mathrm{Cl} \ldots \ldots \\
\text { After pneumothorax: } 200 \mathrm{cc} .\end{array}$ & $\begin{array}{l}20.40 \\
14.53 \\
21.60 \\
21.60 \\
20.65\end{array}$ & $\begin{array}{r}97.6 \\
69.6 \\
103.2 \\
103.2 \\
98.9 \\
\\
100.4\end{array}$ & $\begin{array}{l}65.0 \\
69.5 \\
\\
72.8 \\
71.8 \\
\\
\\
56.0\end{array}$ & \begin{tabular}{|l}
7.60 \\
7.48 \\
7.30 \\
7.34 \\
\\
7.40
\end{tabular} & $\begin{array}{l}29.8 \\
41.4 \\
\\
64.2 \\
58.2 \\
\\
39.7\end{array}$ & $\begin{array}{l}5 \\
6 \\
4 \\
5 \\
2 \\
5 \\
0.5 \\
25\end{array}$ & $\begin{array}{l}270 \\
353 \\
290 \\
\\
353 \\
360 \\
\\
315 \\
300 \\
104\end{array}$ & $\begin{array}{l}1.35 \\
2.11 \\
1.16 \\
1.77 \\
0.72 \\
1.57 \\
0.15 \\
2.59\end{array}$ & $\begin{array}{l}\text { Vagi } \\
\text { intact } \\
\text { through- } \\
\text { out } \\
\text { experi- } \\
\text { ment }\end{array}$ \\
\hline \multirow[t]{2}{*}{19} & 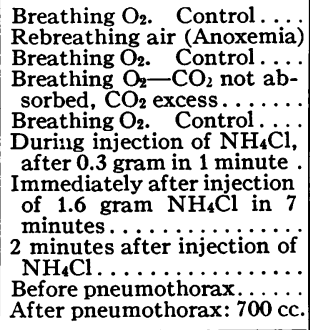 & $\begin{array}{r}18.15 \\
8.28 \\
19.10 \\
18.60 \\
18.36 \\
18.74 \\
17.65\end{array}$ & $\begin{array}{r}98.1 \\
44.8 \\
103.2 \\
100.5 \\
99.3 \\
101.2 \\
95.5\end{array}$ & $\begin{array}{l}57.6 \\
51.4 \\
55.4 \\
57.5 \\
53.2 \\
48.3 \\
\\
37.5\end{array}$ & $\begin{array}{l}7.50 \\
7.58 \\
7.60 \\
7.55 \\
7.63 \\
7.55 \\
7.25\end{array}$ & $\begin{array}{l}32.9 \\
24.6 \\
25.3 \\
\\
29.4 \\
22.7 \\
24.7 \\
\\
37.0\end{array}$ & $\begin{array}{r}20 \\
39 \\
25 \\
\\
39 \\
22 \\
32 \\
\\
\\
\\
36 \\
6\end{array}$ & $\begin{array}{r}155 \\
155 \\
176 \\
270 \\
135 \\
186 \\
\\
\\
128 \\
120 \\
128 \\
93\end{array}$ & $\begin{array}{l}3.10 \\
6.06 \\
4.40 \\
\\
8.10 \\
2.97 \\
\\
5.31 \\
\\
4.62 \\
0.72 \\
0.64 \\
5.42\end{array}$ & $\begin{array}{l}\text { Vagi } \\
\text { intact }\end{array}$ \\
\hline & 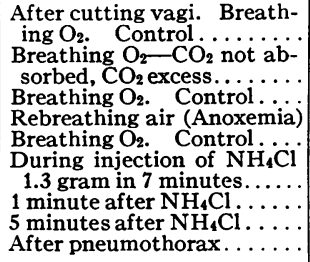 & & & & & & $\begin{array}{r}14 \\
16 \\
12 \\
28 \\
13 \\
15 \\
15 \\
1 \\
2 \\
3\end{array}$ & $\begin{array}{l}288 \\
\\
425 \\
276 \\
363 \\
249 \\
\\
456 \\
360 \\
384 \\
290\end{array}$ & $\begin{array}{r}3.20 \\
6.80 \\
3.31 \\
10.17 \\
3.23 \\
\\
6.85 \\
0.36 \\
0.77 \\
0.87\end{array}$ & Vagi cut \\
\hline \multirow[t]{2}{*}{20} & 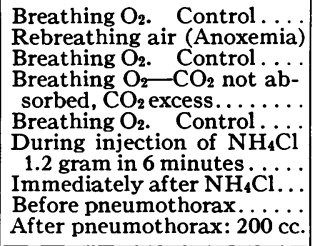 & $\begin{array}{l}19.58 \\
13.20 \\
19.44\end{array}$ & $\begin{array}{r}102.5 \\
69.1 \\
101.7\end{array}$ & $\begin{array}{l}48.9 \\
45.5 \\
47.8 \\
\\
51.6 \\
50.9 \\
\\
46.0\end{array}$ & $\begin{array}{l}7.38 \\
7.65 \\
7.42 \\
\\
7.31 \\
7.46 \\
\\
7.32\end{array}$ & $\begin{array}{l}36.4 \\
18.6 \\
32.5 \\
\\
44.7 \\
31.7 \\
\\
39.0\end{array}$ & $\begin{array}{r}13 \\
29 \\
7 \\
\\
9 \\
10 \\
10 \\
10 \\
3 \\
3 \\
17\end{array}$ & $\begin{array}{l}207 \\
187 \\
320 \\
477 \\
238 \\
\\
274 \\
435 \\
435 \\
207\end{array}$ & $\begin{array}{l}2.70 \\
5.42 \\
2.23 \\
\\
4.30 \\
2.38 \\
\\
2.74 \\
1.30 \\
1.30 \\
3.52\end{array}$ & $\begin{array}{l}\text { Vagi } \\
\text { intact }\end{array}$ \\
\hline & 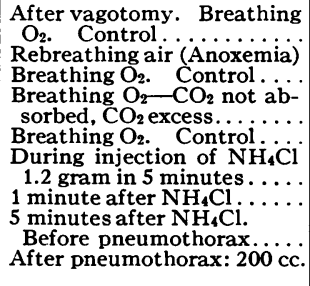 & $\begin{array}{l}15.60 \\
11.64 \\
18.46\end{array}$ & $\begin{array}{l}81.7 \\
60.5 \\
96.8\end{array}$ & $\begin{array}{l}44.7 \\
40.0 \\
45.8 \\
47.9 \\
40.4\end{array}$ & $\begin{array}{l}7.21 \\
7.58 \\
7.31 \\
7.26 \\
7.21\end{array}$ & $\begin{array}{l}48.0 \\
19.1 \\
39.8 \\
49.5 \\
43.4\end{array}$ & $\begin{array}{l}4 \\
6 \\
3 \\
3 \\
4 \\
4 \\
6 \\
1 \\
4 \\
4 \\
6\end{array}$ & $\begin{array}{l}518 \\
621 \\
538 \\
591 \\
497 \\
435 \\
674 \\
436 \\
290\end{array}$ & $\begin{array}{l}2.07 \\
3.73 \\
1.62 \\
1.77 \\
1.99 \\
\\
2.61 \\
0.67 \\
1.74 \\
1.74\end{array}$ & Vagi cut \\
\hline
\end{tabular}


lack was associated with striking decrease in carbon dioxide tension and marked rise in $\mathrm{pH}$. These changes were, of course, the results of the increased ventilation.

Carbon dioxide excess was produced by allowing the animal to rebreathe oxygen from a Benedict spirometer, the soda lime container having been removed. The breathing was more sensitive to carbon dioxide excess than to oxygen lack, the response being not only quantitatively but also qualitatively dissimilar. The reaction to increased carbon dioxide in the inspired air was characterized by relatively greater increase in depth and relatively less increase in rate than was the response to anoxemia.

Although the animals were more sensitive to excess of carbon dioxide than to lack of oxygen, their sensitivity to the former stimulus was much less than has been generally believed. Haldane and Priestley (1905) stated that a rise of one millimeter in the carbon dioxide tension of the alveolar air was sufficient to double the ventilation. Such a change would correspond to a decrease of 0.01 to 0.02 in $\mathrm{pH}$. In our animals the smallest increase in $p \mathrm{CO}_{2}$ found at a time when the ventilation was approximately doubled was $5 \mathrm{~mm}$., and the smallest decrease in $\mathrm{pH}$ was 0.04 . The average change in these values was much greater, being $11 \mathrm{~mm}$. and $0.12 \mathrm{pH}$ respectively when the ventilation was increased by approximately 100 per cent. One might raise the objection that our studies were performed on anesthetized (barbital) dogs, whereas the observations of others have been made on normal men. On the other hand, studies on the alveolar air alone can give at most only incomplete and possibly unreliable information about the blood, even assuming that one gets a true alveolar sample of air. Furthermore, it has already been shown (Cullen and Earle (1929)) that changes of considerable degree may occur in the $\mathrm{pH}$ and $p \mathrm{CO}_{2}$ of normal individuals during the course of the day, with no striking changes in ventilation, and further (Study XIII of this series) that marked changes in ventilation may occur in normal men with no change, or even shifts toward alkalinity, in the $\mathrm{pH}$ and $p \mathrm{CO}_{2}$. For these reasons we agree with Gesell (1927) that the doctrine of the extreme sensitivity of the respiratory center to changes in the blood composition is incorrect.

The $p H$ of the blood was reduced by injecting ammonium chloride. In each experiment characteristic changes in breathing were noted. During the injection the ventilation increased in each instance, this change being due either to increase in depth or rate or both. The change in depth was less striking and less constant than was found when carbon dioxide was breathed, the response to ammonium chloride being similar to that produced by oxygen lack. Within one to three minutes after the injection the breathing decreased in frequency and apnea sometimes resulted. In one animal this was fatal. The $\mathrm{pH}$ was markedly diminished by ammonium chloride in each instance, the degree of decrease varying 
from 0.14 to 0.30 . The carbon dioxide tension was usually elevated when the blood was drawn while the salt was being injected or immediately after. Studies were not made of the blood during the apneic period following the injection but it is considered probable that at this time the $\mathrm{pCO}_{2}$ was much diminished.

In several experiments observations were made, during the apneic period following the administration of ammonium chloride, of the effect of diminishing the vital capacity by pneumothorax. In each instance a prompt increase in respiratory rate was observed, provided the vagi were intact (Fig. 4).

The effect of changes in the acid-base condition of the blood on the breathing of vagotomized dogs was studied in two animals (Table 2, animals 19 and 20). It is seen that oxygen lack, carbon dioxide excess and ammonium chloride were always followed by increased minute ventilation, and of ten followed by increased respiratory rate. Possibly the vagotomized dogs were somewhat less responsive as regards ventilation than were the animals with intact vagi, but our data are not complete enough to prove this. The work of Heymans and Heymans (1927), indicating that not only the respiratory center but the peripheral vagal afferent fibers to the center are also stimulated by chemical changes in the blood, would suggest such a conclusion.

It should be emphasized again that the dogs responded differently to excess carbon dioxide than to oxygen lack or ammonium chloride. According to the Haldane (1922) theory of respiratory control, one would expect a dissimilar reaction to the former two stimuli but one would expect a similar response to acidosis, whether produced by volatile or nonvolatile acid. However, the effect of ammonium chloride was somewhat similar to that of oxygen lack, and was rather unlike that of carbon dioxide excess. This fact seems to confirm the idea of Hooker, Wilson, and Connett (1917), and Scott (1918), that carbon dioxide has a more or less specific effect as well as an acid effect. This specific effect is probably as Jacobs (1920) has suggested, a function of the great diffusibility of $\mathrm{CO}_{2}$. Further observations along this line are being made.

As a result of these observations, showing the relative insensitivity of the ventilation to changes in blood composition, and more especially the relative insensitivity of the rate of breathing to such changes, it seems clear that the marked increases in respiratory rate which were produced by diminishing the vital capacity and which were associated with no change or only slight alterations in the blood composition could not have been due to changes in the blood. These observations, as well as those concerning the effect of vagotomy, seem to show clearly that tachypnea resulting irom diminished vital capacity is due to afferent vagal reflexes. 


\section{DISCUSSION}

In the preceding paper of this series (Calhoun, Cullen, Harrison, Wilkins, and Tims (1931)), studies were made of orthopnea. It was found, as Christie and Beams (1922) had previously reported, that on assuming the recumbent posture the patient with orthopnea has a significant reduction of vital capacity. Hence, in the recumbent posture the tidal air became a greater fraction of the vital capacity and this was believed to be one important cause of the dyspnea in the recumbent position. But it was also found that patients with severe orthopnea of ten breathed more rapidly in the recumbent than in the sitting posture, a paradoxical effect, being the opposite reaction to that usually observed in normal persons who of ten breathe more slowly in the recumbent position. It was evident that this increase in the rate of breathing in a person whose respiratory muscles were already performing more than the normal amount of work was an important factor in the production of further subjective respiratory distress. In the study referred to, the cause of this increase in the rate of breathing was not found. The present study indicates clearly that decrease in vital capacity can, per se, produce rapid breathing by reflexes from the lungs. The mechanism of orthopnea therefore seems to be clear.

The patient with congestive heart failure of moderate degree has a considerable decrease in his vital capacity, even when sitting. Although his respiratory rate is likely to be somewhat greater than normal his tidal air is usually a larger fraction of his vital capacity than is the case in a normal individual. This in itself means a greater respiratory effort per breath. But, due to his decreased vital capacity he has a slight increase in respiratory rate. Hence, he has to make more than the normal number of muscular efforts per minute to breathe. Because of the great reserve power of the respiratory muscles he may have only slight or even no respiratory distress.

On lying down his vital capacity is, because of increased volume of blood in the lung and higher diaphragm, diminished still further, by possibly 300 cubic centimeters. To a normal subject such a decrease, being only perhaps eight per cent of the total vital capacity, would make no perceptible difference. But as has been stated, the patient was already unconsciously drawing on his respiratory reserves, and furthermore, this decrease may represent a fifteen per cent (or greater) diminution in his vital capacity. If his minute ventilation is to remain the same he has to either breathe a still larger fraction of his vital capacity at each breath or he has to breathe faster. Actually he does both in many cases. The result is not only an increase in the muscular effort involved for each breath but, because of vagal reflexes, an increase in the number of efforts each minute. He was already using some of the reserve power of his respiratory muscles but not enough to be conscious of the effort involved. 
The increase in the muscular effort of breathing becomes, on lying down, sufficient to produce a sensation of fatigue in the respiratory muscles and he feels "short of breath." Other factors, such as the sense of congestion in the head due to the increase in venous pressure on lying down and slight decrease in the aeration of the blood in the recumbent posture, may play some rôle but the essential features in the mechanism of his orthopnea are those described.

At a later stage of his disease, the patient's vital capacity is still less. Even when he sits up it is very low. The result is the constant resting dyspnea, which is such a distressing feature of the terminal stage of cardiac failure in many patients.

Orthopnea occurs not only in cardiac disease but also in certain other conditions, such as severe cases of pneumonia, pleural effusion, marked ascites, pneumothorax, massive collapse of the lung, etc. All of these conditions are characterized by diminished vital capacity and by increased respiratory rate. It seems to us likely that in these cases also orthopnea and dyspnea are due, in the main, to decreased vital capacity and consequent reflex tachypnea. Dyspnea in pneumonia has never been satisfactorily explained. Undoubtedly, pain, fever with increased metabolic rate, and anoxemia play a rôle. However, one observes patients with pneumonia who have little or no pain, whose anoxemia has been relieved by oxygen therapy, and who still are breathing rapidly-more rapidly than can be explained by fever alone. It is probable that the rapid breathing in such cases is due to reflexes caused by decrease in vital capacity. This conclusion is somewhat similar to that arrived at by Binger and his co-workers (1925-1927), who in a series of studies on rapid breathing were led to the view that the changes in the lungs themselves were responsible for the tachypnea.

Investigations of the blood have provided satisfactory explanations for the breathing of diabetic and renal acidosis, which are characterized by marked increase in the depth and relatively slight increase in the rate of breathing. Adequate explanations for the shallow rapid breathing of conditions associated with cardiac or pulmonary disorders and hence with decreased vital capacity, have not been furnished by chemical studies of the blood, and hitherto have been lacking. We believe that reflexes from the lungs are the chief cause of dyspnea in such conditions.

The concept of the extreme delicacy of the respiratory response to chemical alterations in the blood must, as has been emphasized by Gesell (1927), be abandoned. From the present and the preceding studies of this series (XIII, Cullen et al.; XIV, Calhoun et al.) it seems evident that the nervous regulatory mechanism is more delicate than the chemical. One can think of the latter as being like the coarse adjusting screw and of the former as being like the fine adjusting screw of a microscope. The sensitivity of the reflex respiratory control is a fact of considerable signifi- 
cance. Because of it respiration is altered in such a way as to tend to prevent gross chemical changes in the blood. The constancy of the "milieu intérieur" (Claude Bernard) is thereby maintained.

\section{SUMMARY}

Studies have been made of the respiratory rate and depth, the minute ventilation, and of the oxygen, carbon dioxide and $\mathrm{pH}$ of the arterial blood and of the venous blood from the brain of dogs anesthetized with barbital. In some experiments artificial reduction of vital capacity was produced either by pneumothorax, by introducing fluid into the lungs through the trachea, or by distending the capillaries of one lung with blood, according to a technique which has been described. In other experiments observations were made concerning the sensitivity of the respiration to oxygen lack, carbon dioxide excess and to acidosis produced by the intravenous injection of ammonium chloride. The following results were obtained.

1. Reduction of vital capacity by any of the methods used resulted in rapid breathing, provided the vagus nerves were intact. In such experiments chemical changes of the blood were usually either absent or in the direction of increased alkalinity.

2. In vagotomized dogs diminution in vital capacity was usually not followed by rapid breathing, unless the diminution was of sufficient degree to produce either marked oxygen lack or increased acidity of the blood.

3. Oxygen lack, produced by rebreathing, caused increased ventilation either by increase in depth, rate or both. In order to double the ventilation it was usually necessary that the arterial blood be less than 60 per cent saturated.

4. Carbon dioxide excess caused marked increase in depth and relatively slight increase in rate of breathing. The response of the animals to carbon dioxide excess was quantitatively greater and qualitatively different from that of oxygen lack. In order to double the ventilation it was usually necessary to produce a fall of approximately $0.10 \mathrm{in} \mathrm{pH}$ and a rise of $10 \mathrm{~mm} . \mathrm{Hg}$ in carbon dioxide tension of the arterial blood.

5. The effect of the acidosis produced by ammonium chloride on the breathing was unlike that of carbon dioxide excess and rather similar to that of oxygen lack, being characterized by a relatively great increase in rate and only slight increase in depth. Following the injection of ammonium chloride apnea sometimes occurred.

6 . Chemical changes in the blood never produced the extreme degree of tachypnea which resulted from dininished vital capacity.

7. Vagotomized dogs, although insensitive to diminution in vital capacity, reacted with increase in ventilation to chemical changes in the blood. 


\section{CONCLUSIONS}

From these observations the following conclusions have been drawn.

1. Orthopnea and the continuous dyspnea at rest which occurs in the terminal stages of cardiac disease are of reflex origin and dependent on diminished vital capacity.

2. It is probable that the rapid breathing found in various diseases of the thoracic organs accompanied by decrease in vital capacity is essentially of reflex origin.

3. The reflex mechanism of respiratory control is more sensitive than the chemical mechanism. The respiratory center seems to be much less sensitive to alterations in the composition of the blood than has been generally believed.

\section{BIBLIOGRAPHY}

Binger, C. A. L., Boyd, D., and Moore, R. L., J. Exp. Med., 1927, xlv, 643. The Effect of Multiple Emboli of the Capillaries and Arterioles of One Lung.

Binger, C. A. L., Brow, G. R., and Branch, A., J. Clin. Invest., 1925, i, 127. Experimental Studies on Rapid Breathing. I. Tachypnea Independent of Anoxemia Resulting from Multiple Emboli in the Pulmonary Arterioles and Capillaries.

Binger, C. A. L., Brow, G. R., and Branch, A., J. Clin. Invest., 1925, i, 155. Experimental Studies on Rapid Breathing. II. Tachypnea Dependent upon Anoxemia Resulting from Multiple Emboli in the Larger Branches of the Pulmonary Artery.

Binger, C. A. L., and Moore, R. L., J. Exp. Med., 1927, xlv, 633. Changes in Carbon Dioxide Tension and Hydrogen Ion Concentration of the Blood Following Multiple Pulmonary Embolism.

Calhoun, J. A., Cullen, G. E., Harrison, T. R., Wilkins, W. E., and Tims, M. M., J. Clin. Invest., 1931, x, 833. Studies in Congestive Heart Failure. XIV. Orthopnea: Its Relation to Ventilation, Vital Capacity, Oxygen Saturation, and Acid-Base Condition of Arterial and Jugular Blood.

Christie, C. D., and Beams, A. J., Arch. Int. Med., 1922, xxx, 34. The Estimation of Normal Vital Capacity with Especial Reference to the Effect of Posture.

Cullen, G. E., J. Biol. Chem., 1922, lii, 501. Studies of Acidosis. XIX. The Colorimetric Determination of the Hydrogen Ion Concentration of Blood Plasma.

Cullen, G. E., and Earle, I. P., J. Biol. Chem., 1929, 1xxxiii, 545. Studies of the Acid-Base Condition of Blood. II. Physiological Changes in AcidBase Condition Throughout the Day.

Cullen, G. E., Harrison, T. R., Calhoun, J. A., Wilkins, W. E., and Tims, M. M., J. Clin. Invest., 1931, x, 807. Studies in Congestive Heart Failure XIII. The Relation of Dyspnea of Exertion to the Oxygen Saturation and Acid-Base Condition of the Blood.

Gesell, R., Physiol. Rev., 1927, v, 551. The Chemical Regulation of Respiration.

Haldane, J. S., Respiration. Yale University Press, New Haven, 1922.

Haldane, J. S., and Priestley, J. G., J. Physiol., 1905, xxxii, 225. The Regulation of the Lung-Ventilation. 
Harrison, T. R., and Moore, R. L., Ztschr. f. d. ges. exp. Med., 1928, 1xi, 305. Über die Beziehungen des $\mathrm{N}$. vagus zum Atmungsmechanismus.

Harrison, T. R., Turley, F. C., Jones, Edgar, and Calhoun, J. A., Arch. Int. Med., 1931, xlviii, 377. Studies in Congestive Heart Failure. X. The Measurement of Ventilation as a Test of Cardiac Function.

Herring, E., and Brauer, J., Sitzungsb. d. Akad. Wien, Mathem-.naturw., 1868, lviii, 909.

Hertzman, A. B., and Gesell, R., Am. J. Physiol., 1927, lxxxii, 608. The Regulation of Respiration. XII. The Vagal Reflex Control of the Respiratory Movements of the Isolated Head.

Heymans, J. F., and Heymans, C., Arch. Internat. de Pharmacodyn. et de Therap., 1927, xxxiii, 273. Sur les Modifications Directes et sur la Régulation Réflexe de l'Activité du Centre Respiratoire de la Tête Isolée du Chien.

Hooker, D. R., Wilson, D. W., and Connett, H., Am. J. Physiol., 1917, xliii, 351. The Perfusion of the Mammalian Medulla: The Effect of Carbon Dioxide and Other Substances on the Respiratory and Cardio-vascular Centers.

Jacobs, M. H., Am. J. Physiol., 1920, li, 321. To What Extent are the Physiological Effects of Carbon Dioxide Due to Hydrogen Ions?

Jacobs, M. H., Am. J. Physiol., 1920, liii, 457. The Production of Intracellular Acidity by Neutral and Alkaline Solutions Containing Carbon Dioxide.

Legallois, C. J. J., Expériences sur le principe de la Vie. Paris, 1812.

Moore, R. L., J. Exp. Med., 1927, xlvi, 819. A Study of the Hering-Breuer Reflex.

Pilcher, C., Anat. Rec., 1930, xliv, 363. A Note on the Occipito-Vertebral Sinus of the Dog.

Scott, R. W., Am. J. Physiol., 1918, xlvii, 43. The Significance of Undissociated Carbon Dioxide in Respiration.

Van Slyke, D. D., and Neill, J. M., J. Biol. Chem., 1924, 1xi, 523. The Determination of Gases in Blood and Other Solutions by Vacuum Extraction and Manometric Measurement.

Van Slyke, D. D., and Sendroy, J., Jr., J. Biol. Chem., 1927, lxxiii, 127. Carbon Dioxide Factors for the Manometric Blood Gas Apparatus. 